\title{
First eighteen months of simultaneously measurements of the energy spectrum of Cosmic- Ray induced neutrons on the Pic-du-Midi Observatory and the Concordia Station in Antarctica
}

\author{
Guillaume HUBERT ${ }^{1}$ \\ ONERA, the French Aerospace Lab. \\ 2, avenue Edouard Belin, 31055, Toulouse \\ E-mail: guillaume.huberteonera. fr
}

A neutron spectrometer network composed by two high-altitude stations was operated by the French Aerospace Lab. And using Bonner Sphere System extended to high energies. This instrument can measure the neutron spectrum over a wide energy range from meV up to tens of $\mathrm{GeV}$ with a short time resolution, allowing to investigate solar flare. Instruments are operated in the Pic-du-Midi (French Pyrenees, +2885m) since May 2011 and, in the framework of the CHINSTRAP project supported by the IPEV (the French Polar Institute) in Concordia station (Antarctica, $+3233 \mathrm{~m}$ ) since December 2015, respectively. In this work, first eighteen months of simultaneously measurements of the energy spectrum of indirect-CR are presented and analysed. In addition to the usual analyses (i.e. count rates and fluxes), neutron spectrum and their energetic domain are investigated. Spectrum measurements are coupled with transport modelling including simulation of atmospheric cascades and primary spectra which only depend on the solar modulation potential (i.e. Force-Field Approximation). Thus, a part is devoted to study the solar modulation potentials extracted from the both stations. Results show that is possible to characterize quantitatively the neutron spectrum in the Pic-du-Midi using modelling and cascade neutron measured in Concordia (and reciprocally). Contributions induced by cascade neutrons can be extracted from measurements to deduce the solar potential, allowing to deduce or to enrich indirect-CR specifications for other locations and conditions. An applied case is presented for the Antarctic continent and it compares and to cross-analyses the indirectCR variations from the neutron spectrum and NMs during 2016.

35th International Cosmic Ray Conference - ICRC2017

10-20 July, 2017

Bexco, Busan, Korea

1

Speaker

(C) Copyright owned by the author(s) under the terms of the Creative Commons 


\section{Introduction}

The primary and the secondary radiation produced in the atmosphere can be a serious issue for the reliability of microelectronics devices [1]-[4] and this is of a major concern for aircrew member's dose assessment [5]. Moreover, Polar Region development induces an important issue related to space weather. The main secondary particles produced by the interaction of primary cosmic rays (CR) with the nuclei of the constituents of the atmosphere are neutrons, protons, muons, pions and electrons [6].

To characterize the indirect-CR, many neutron detectors are distributed around the earth, especially in high latitudes. The Neutron Monitors (NMs) are used to measure and record the count rates induced at ground level by secondary radiation generated by atmospheric cascades. Currently, more than 50 neutron monitor stations distributed [7], including several in polar region as Amundsen-Scott and Mc Murdo stations [8]. CR induced neutrons are the main contribution $(95 \%)$ in the NM count rates, but secondary muons and protons represents a significant amount that must be considered. NMs provide intensity on the relative variation of the primary $\mathrm{CR}$, however various techniques related to the multiplicity or details on the timing of neutrons originating in the lead allow spectral resolution. Recently, a neutron spectrometer network composed by two high altitude stations was developed by the French Aerospace Lab thanks to an advanced BSS extended to high energies [9]. This instrument can measure the neutron spectrum over a wide energy range from meV up to tens of $\mathrm{GeV}$ with a short time resolution allowing to enrich analyses of CR.

Thus, this paper presents the first eighteen months of simultaneously measurements of the energy spectrum of CR induced neutrons on the both stations and their analyses. A first part is devoted to analyze the count rates, the spectrum and the neutron fluxes, implying crosscomparisons. In a second part, measurements are coupled to transport simulations based on atmospheric cascades modelling according to primary spectra which only depend on the solar modulation potential [10]. This parameter is investigated from coupled cascade neutron measurements, demonstrating the interest to extrapolate a global indirect CR approach. Thus, contributions induced by cascade neutrons can be extracted from measurements to deduce the solar potential, allowing to deduce or to enrich indirect-CR specifications for other locations and conditions. An applied case is presented for Antarctic, it compares the indirect-CR variations from the neutron spectrum and NMs during the 2016 year.

\section{Experimental platform and modeling}

\subsection{Instrument descriptions}

A Bonner multi-sphere neutron spectrometer extended to high neutron energies was developed to measure the energy spectrum of the CR induced neutrons, considering the energy range from $\mathrm{meV}$ to $\mathrm{GeV}$ [11]. As detailed in previous works, this system was composed of spherical $3 \mathrm{He}$ proportional counters surrounded with spherical PEHD (high density polyethylene) moderators with different thicknesses. Additionally, the spectrometer includes 
two PEHD spheres with inner tungsten and lead shells (7/8" and 9", respectively) in order to increase the response to neutrons above $20 \mathrm{MeV}$. Electronic part dedicated to drive each signal, was composed to the pre-amplifier and the linear amplifier. The output pulse signals are treated by a multi channel analyzer, translated as spectrum or count. The total counts of each detector were obtained by summing the total counts over a given integration time. The response functions (deduced from GEANT4 and/or MCNPx calculations) were used to convert the measured counting rates to neutron energy spectrum.

\subsection{High Altitude Stations and platform descriptions}

Since May 2011, the French Aerospace Lab operated a neutron spectrometer at the summit of the Pic-du-Midi [9] (+2885m above sea level), and in the framework of the CHINSTRAP project support by IPEV (French Polar Institute). A second neutron spectrometer is operated in the Concordia research station (Antarctica) since December 2015. This station is located at Dome $\mathrm{C}$ on the Antarctic Plateau $\left(75^{\circ} 06^{\prime} \mathrm{S}, 123^{\circ} 23^{\prime} \mathrm{E}\right)$ at $3233 \mathrm{~m}$ above the sea level. This instrument significantly expands the capability of the neutron spectrometer operated in the Piddu-Midi because the site has an almost zero rigidity cut-off $(\mathrm{R}<0.01 \mathrm{GV})$, i.e., no geomagnetic shielding even for low-energy particles. Thus, each energy, even the lowest coming from specific solar events, are able to penetrate in the atmosphere. Fig. 1 presents the Concordia station, the Pic du Midi Observatory and their neutron spectrometers. The altitude, latitude, longitude, cut-off rigidity and atmospheric depth are given in the complementary table.
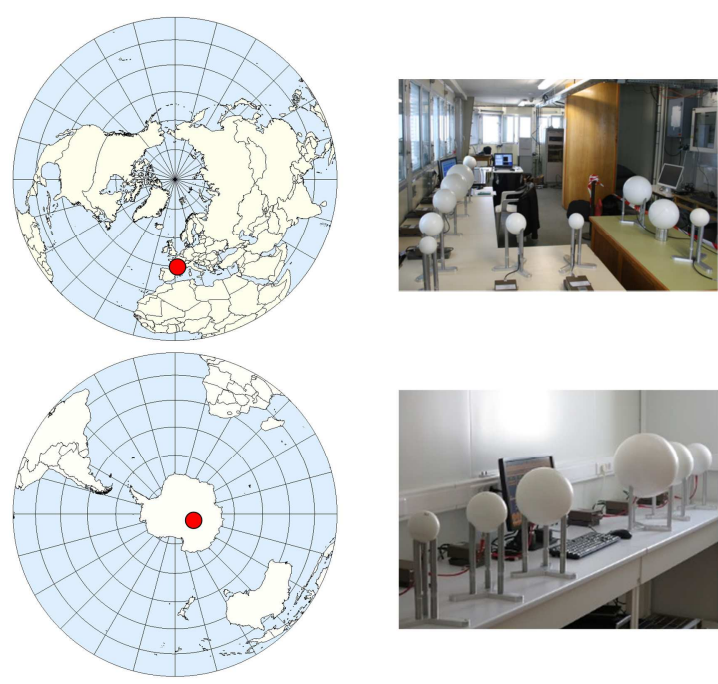

\begin{tabular}{lll}
\hline & $\begin{array}{l}\text { Pic-du-Midi, } \\
\text { France }\end{array}$ & $\begin{array}{l}\text { Concordia } \\
\text { station, } \\
\text { Antarctica }\end{array}$ \\
\hline $\begin{array}{l}\text { ACRONYM } \\
\text { (Lab., project) }\end{array}$ & ACROPOL & CHINSTRAP \\
$\begin{array}{l}\text { Altitude } \\
\text { Latitude }\end{array}$ & $\begin{array}{l}2885 \mathrm{~m} \\
42^{\circ} 55^{\prime} \mathrm{N}\end{array}$ & $\begin{array}{l}3233 \mathrm{~m} \\
75^{\circ} 06^{\prime} \mathrm{S}\end{array}$ \\
Longitude & $0^{\circ} 08^{\prime} \mathrm{E}$ & $123^{\circ} 19^{\prime} \mathrm{E}$ \\
\hline Cut-off rigidity & $5.6 \mathrm{GV}$ & $<0.001 \mathrm{GV}$ \\
\hline $\begin{array}{l}\text { Atmospheric } \\
\text { depth }\end{array}$ & $700.7 \mathrm{~g} / \mathrm{cm}^{2}$ & $635 \mathrm{~g} / \mathrm{cm}^{2}$ \\
\hline $\begin{array}{l}\text { Neutron } \\
\text { experiment }\end{array}$ & Neutron spectrometer \\
\hline
\end{tabular}

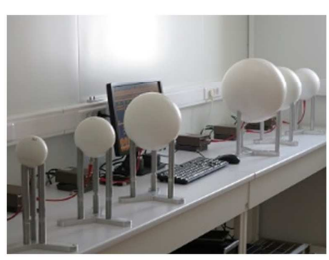

$\begin{array}{lll}\text { Start Operating } \quad \text { May 2011 } & \text { December } \\ 2015\end{array}$

Fig. 1. View of the scientific shelter (Concordia station) and the neutron spectrometer operated since December 2015 (altitude, latitude and longitude are given in table I).

\subsection{Modeling approach based on previous works}

The Force-Field approximation model provides a simple parametric approximation of the differential spectrum of GCR. It contains only one variable parameter and, therefore, the whole 
energy spectrum (in the energy range from $100 \mathrm{MeV} /$ nucleon to $100 \mathrm{GeV} /$ nucleon) for protons and $\alpha$-particles can be described by a single number, the modulation potential $\phi(t)$ whose value is given in units of MV, within the framework of the adopted local interstellar spectrum. The modulation potential $\phi(\mathrm{t})$ is only a formal spectral index whose physical interpretation is not straightforward, especially on short time scales and during periods of active Sun. Virtually, everything is somehow correlated to overall solar activity, and some parameters like $\phi(t)$ and sunspot number are particularly relevant at providing an overview. Thus, several strategies have been developed for the reconstruction of time series of the modulation potential $\phi(t)$. Among these methods, an atmospheric radiation model named ATMORAD [10] based on GEANT4 simulations and Force-Field Approximation, allows to evaluate the modulation potential.

\section{First eighteen months of simultaneously measurements of neutron spectrum}

To illustrate the atmospheric pressure impact, Fig. 2 presents the counts integrated during 5 minutes on 9" $\mathrm{Pb} / \mathrm{PEHD}$ detector operated in the Concordia station and the Pic-du-Midi Observatory as function of the atmospheric pressure.

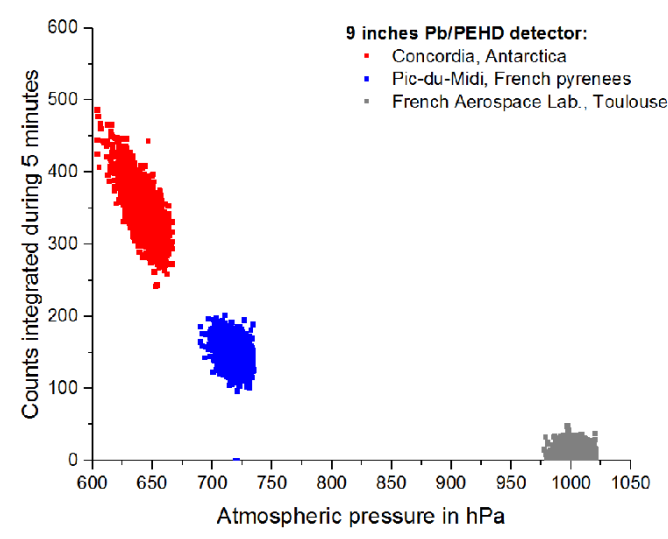

Fig. 2. Counts integrated during 5 minutes for 9" $\mathrm{Pb} / \mathrm{PEHD}$ detector operated in the Concordia station, the Pic-du-Midi observatory and in the Toulouse city, as function of the atmospheric pressure. Data were registered from December 2015 to May 2017.

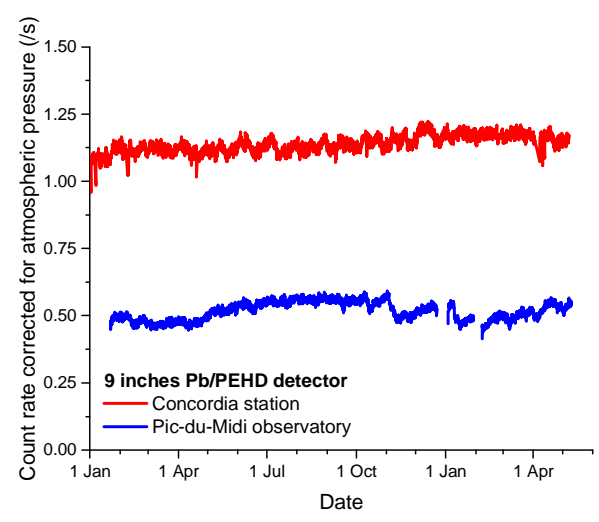

Fig. 3. Count rate registered by the neutron spectrometer operated in the Concordia station and the Pic-du-Midi observatory from January 2016 to May 2017 (quiet period), for the 9" lead metallic detectors. The count rates are corrected for atmospheric pressure and the integration time is considered as 5 minutes.

CR quantities increase at higher altitude as air pressure and the shielding effect of the atmosphere derease. More precisely, the vertical distribution of temperature, pressure, density and composition of the atmosphere constitute the atmospheric structure, and these quantities also vary with season and location in latitude and longitude, as well as from night to day. However under the topic of atmospheric structure, the focus is on the average variations with height above sea level. Then, the meteorological data such as temperature, air pressure and relative humidity are measured simultaneously every five seconds by three sensors.

Fig. 3 presents the count rate corrected for the atmospheric pressure for the Concodia station and the Pic-du-Midi observatory, considering the 9" lead metallic detectors and a integration time of 5 minutes. The reference atmospheric pressure level is set to 650 and 725 
mbar respectively for Concordia and Pic-du-Midi. Data recorded were performed during quiet solar activity. Except a proportionality factor, trends are fairly similar between the two stations, count rates are 1.1 and 0.5 count per second in the Concordia and Pic-du-Midi stations, respectively. An oscillation is perspirable on Pic-du-Midi data, mainly induced by the winter/summer cycles i.e. a process of neutron attenuation and thermalization induced by snow.

The CR neutron energy spectra can be obtained by unfolding the measured counting rates using response function, deconvolution method (i.e. GRAVEL) and considering initial spectrum (neutron spectrum at sea level observed by Goldhagen et al. [12]). Fig. 4 presents the atmospheric neutron spectrum in lethargic representation, for the Pic-du-Midi observatory and the Concordia station during the period from December 2015 to May 2017.

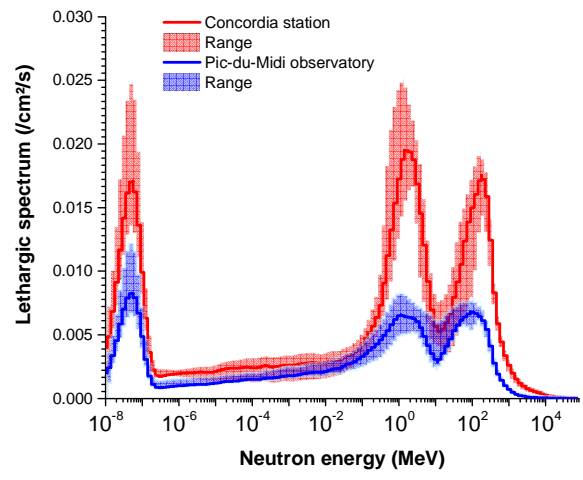

Fig. 4. Atmospheric neutron spectrum in lethargic representation obtained in the Pic-du-Midi observatory and the Concordia station during the period from December 2015 to May 2017.

Fig. 5 presents the integrated fluences rates derived from measurements in the both station during the period from December 2015 to May 2017. Contributions induced by cascade neutrons is extracted from results. Concerning the Pic-du-Midi measurments, a significant temporal oscillation is observed for total integrated fluence rates, mainly induced by snow thickness during winter. As shown in previous works, seasonal oscillations are governed by ground albedo neutron impacts on thermal, epithermal and evaporation domains. Conversely, no oscillation is observed for Concordia measurements, due to the stability of the meteoroligal environment (i.e. a few centimeters of snow per year). The solar potential $\varphi(t)$ is directly linked to the Sun's Activity. Moreover, as illustrated in Fig. 5, cascade neutrons were lowly impacted by the hydrogen density variations, allowing their uses to deduce the solar potential $\varphi(t)$. Thus, Fig. 6 shows the solar potential dynamics deduced from measurements operated in the both stations during 18 month (from December 2015 to May 2017). The integration time is considered equal to 1 day. Orders of magnitude are consistent, demonstrating the ability to link simultaneous measurements. In 2015, a similar consistence was obtained considering the Picdu-Midi and Pico dos Dias measurements [9]. Measurements can be quantitatively correlated using modeling and cascade neutron in quiet solar activity. It is likely that this conclusion is nuanced in the case of solar events, particularly during a GLE event. 

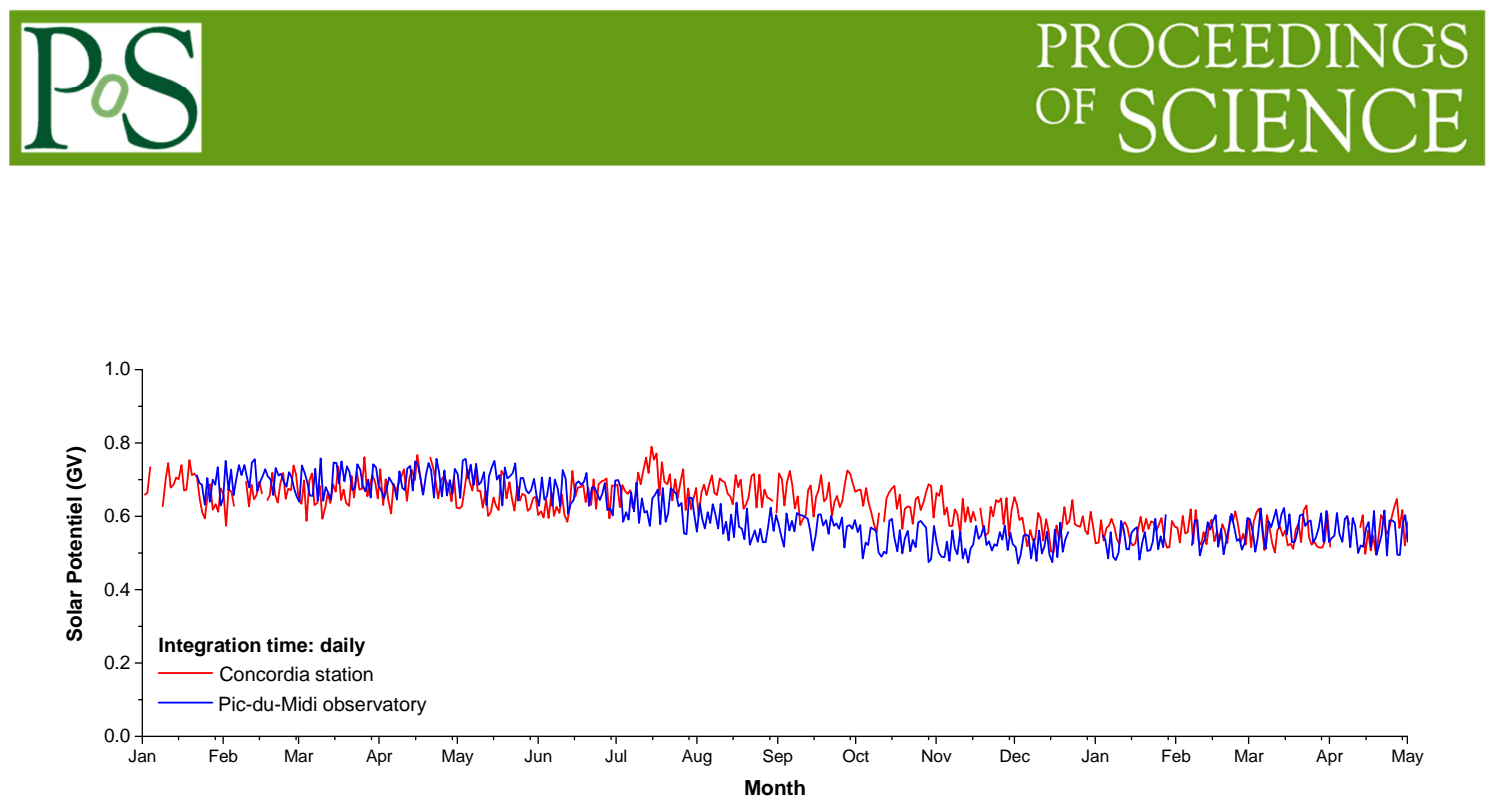

Fig. 6. Atmospheric neutron spectrum in lethargic representation obtained in the Pic-du-Midi observatory and the Concordia station during the period from December 2015 to May 2017.

\section{Cross comparison with NMs operated in Antarctica}

Bartol Research Institute currently operates height NMs including two in Antarctica, in Mac Murdo and Amundsen-Scott stations since 1957 and 1964, respectively [13]. Thus, NM real-time data are available and they can be compared dynamically. Moreover, knowing the measured neutron spectrum at Concordia and NM response functions, it is possible to deduce the equivalent NM count rate. Muon and proton contributions can be considered by extrapolating their spectra from measured cascade neutron and ATMORAD. Equivalent NM count rate $C_{N M}$ calculation in Concordia station is described by the equation (1):

$$
C_{N M}=\int \eta_{n}(E) \cdot \frac{\partial \varphi_{n, \text { exp }}(E)}{\delta E} \cdot d E+\sum_{i=\text { proton, }, \text { mon }} \int \eta_{i}(E) \cdot \frac{\partial \varphi_{i, \text { ATMO }}\left(E, \phi_{\text {ATMO }}\left(n_{\text {cas }}\right)\right)}{\delta E} \cdot d E
$$

Where $\eta_{i}(E)$ are the NM response functions ( $\mathrm{i}=$ neutron, proton, muon), $\partial \varphi_{n, \text { exp }}(E) / \delta E$ is the neutron spectrum deduced from the neutron spectrometer data and $\partial \varphi_{\text {АтмO }}\left(E, \phi_{\text {AтMO }}\left(n_{\text {саa }}\right)\right) / \delta E$ are the proton and muon spectrum calculated thanks to ATMORAD and the solar potential deduced from neutron cascade fluxes. Fig. 7 compares count rate dynamics from January 2016 to 2017 registered by the NM in the Mac Murdo and the Amundsen-Scott stations, and equivalent NM count deduced from the neutron spectrometer in Concordia. Cosmic ray levels have been steadily increasing in recent months, which reflects the descending part of the solar cycle. It is interesting to note that these results illustrate the possibility to couple analyzes from different instruments.

Moreover, this can be extended to equivalence NM response calculations applied to other locations, taking into account the realistic altitude, thanks to the equation (2):

$$
C_{N M}(\text { lat., long., alt. })=\sum_{i=n, p, \mu} \int \eta_{i}(E) \cdot \frac{\partial \varphi_{i, A T M O}\left(E, \phi_{A T M O}\left(n_{\text {cas }}\right), \text { lat., long., alt. }\right)}{\delta E} d E
$$


Fig. 8 presents a cartography of the count rate (in unit count /hour/100 per ${ }^{3} \mathrm{He}$ tube) recorded in July 2016. Results are issued from neutron spectrometer measurements in Concordia and ATMORAD to extrapolate for Antarctic continent. Ground altitudes, considering rock and ice thickness, are issued to Quantarctica tool [14]. In this geographical area, the altitude is the main parameter on the cosmic ray level, since the influence of the magnetic field is low.

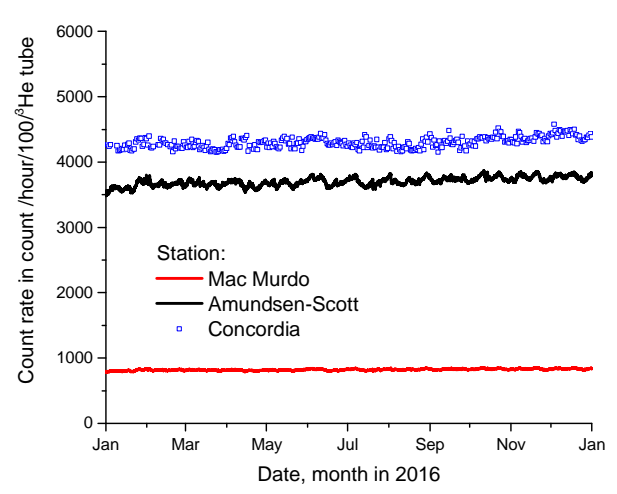

Fig. 7. Count rate (in count /hour/100 per ${ }^{3} \mathrm{He}$ tube) from January 2016 to 2017 registered by the NM in the Mac Murdo and the Amundsen-Scott stations, and equivalent NM count deduced from the neutron spectrometer in Concordia

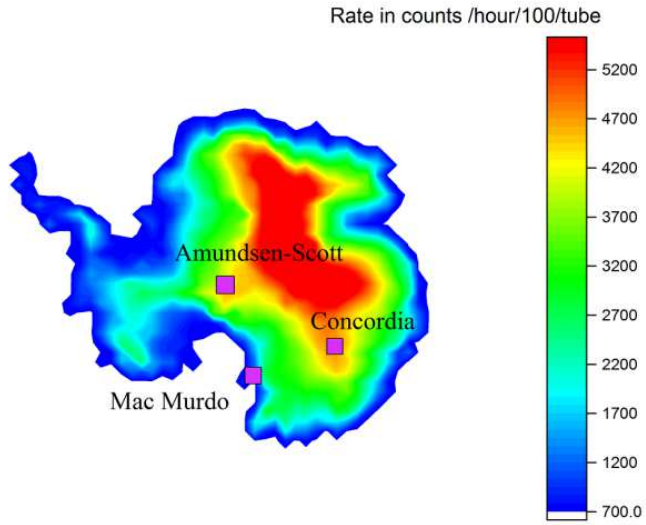

Fig. 8. Cartography of the count rate (in count /hour/100 per ${ }^{3} \mathrm{He}$ tube) recorded in July 2016. Results are issued from neutron spectrometer measurements in Concordia and ATMORAD to extrapolate for Antarctic continent.

\section{Conclusion}

First eighteen months of simultaneously measurements of the energy spectrum of CR on the Pic-du-Midi Observatory and the Concordia Station in Antarctica are presented. In addition to the usual analyzes of count rates and fluxes, neutron spectrometers allow to investigate the neutron spectrum and energetic domain dynamics. The dispersion of the spectrum is relatively significant, but mainly due to variations in the absolute CR level. Moreover, energetic fluctuations are more significant in the thermal, epi-thermal and evaporation domains.

Contributions induced by cascade neutrons are extracted from measurements to deduce the solar potential, which in quiet solar period allows to deduce or to enrich CR specifications for other locations and conditions. Applied in the particular case of the Antarctic continent, it is possible to compare and to cross-analyze the CR dynamics from the neutron spectrum and NMs.

\section{Acknowledgments}

The authors thank F. Lacassagne and the technical staff for their supports at the Pic-duMidi observatory. We are thankful to the personnel of the Concordia station for their great help and hard work in harsh conditions, D. Tuillier from IPEV, L. Milano and S. Chicarella.

The Bartol Research Institute neutron monitor program is supported by the United States National Science Foundation under grants PLR-1245939 and PLR-1341562, and by the University of Delaware Department of Physics and Astronomy and Bartol Research Institute.

Quantarctica was originally developed for in-house use at the Norwegian Polar Institute. 


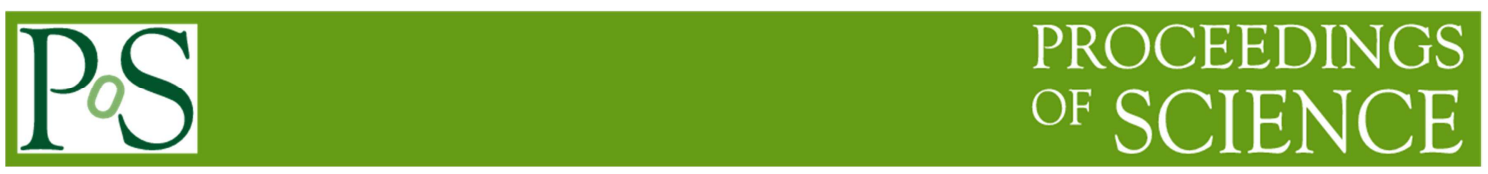

\section{References}

[1] P. Peronnard et al., Nuclear Science, IEEE Transactions on, 56(6):3450 - 3455, 2009.

[2] G. Hubert et al., Integration, the VLSI Journal, 50: 39-47, 2015

[3] D. Lambert et al., Nuclear Science, IEEE Transactions on, 53(4):1890 - 1896, 2006.

[4] G. Hubert at al., Nuclear Science, IEEE Transactions on, 60(4):2418-2426, 2013.

[5] G. Reitz, Radiat Prot Dosimetry, 1993; 48(1):5-20.

[6] P. K. F. Grieder, 1st ed., pp. 1093, Elsevier, 2001.

[7] Neutron Monitor DataBase, available: http://www.nmdb.eu.

[8] J. Bieber et al., Journal of Geophysical Research: Space Physics, 2013; 118: 6847-6851.

[9] G. Hubert et al., Astroparticles Physics, 74: 27-36, 2016.

[10] G. Hubert et al., Radiation Research, 184(1): 83-94, 2015.

[11] A. Cheminet et al., IOP Science Journal of Instr., 2nd FNDA, Vol. 7, 2012.

[12] P. Goldhagen, MRS Bulletin, 28(2):131 (2003).

[13] Univ. of Delaware, Bartol Res. Institute Neutron Monitor program, http://neutronm.bartol.udel.edu/

[14] Quantarctica, collection of Antarctic geographical datasets, available: http://quantarctica.npolar.no/ 